\title{
AOR
}

Selected Papers of \#AolR2018:

The $19^{\text {th }}$ Annual Conference of the

Association of Internet Researchers

Montréal, Canada / 10-13 October 2018

\section{MO' CHARACTERS MO' PROBLEMS: ONLINE SOCIAL MEDIA PLATFORM CONSTRAINTS AND MODES OF COMMUNICATION}

Lewis Mitchell

School of Mathematical Sciences, The University of Adelaide, SA 5005, Australia

Joshua Dent

School of Mathematical Sciences, The University of Adelaide, SA 5005, Australia

Joshua V Ross

School of Mathematical Sciences, The University of Adelaide, SA 5005, Australia

\section{Introduction}

This paper explores a foundational change to one of the world's largest social media platforms: Twitter's doubling of its character limit to 280 characters on 7 November 2017. This arguably represents the most significant change to an individual's everyday experience of this platform since its inception in 2006. Twitter argued that this change made it "easier to tweet", with individuals "less time editing their Tweets in the composer" and that the "timeline reading experience should not substantially change" (Rosen 2017). We test these claims quantitatively, by applying natural language processing techniques as well as mathematical modeling approaches to a large dataset collected before and after the change. Our results show that the average timeline reading experience did in fact change significantly after the change, and reveal a surprisingly long "reaction time" of the Twitter-using population to this exogenous change to the communication medium. We postulate that this behavior is rooted in human psychology, and explain how our findings are consistent with previous experimental studies of mental perceptions of text lengths.

\section{Data collection and methodology}

We analysed an existing dataset comprising 540 million English-language tweets (after filtering as below), collected using Twitter's decahose feed (10\% of all public tweets) between 1 September 2017 and 12 January 2018. To focus on the change in behavior by individuals when writing tweets we filtered out all tweets containing URLs, and in order to apply natural language processing techniques we considered only English-

Suggested Citation (APA): Mitchell, L. (2018, October 10-13). Mo' characters mo' problems: Online social media platform constraints and modes of communication. Paper presented at AolR 2018: The 19 $19^{\text {th }}$ Annual Conference of the Association of Internet Researchers. Montréal, Canada: AolR. Retrieved from http://spir.aoir.org. 
language tweets (as determined by Twitter's automatic language detection algorithm and stored in the metadata for each tweet).

To test whether the "timeline reading experience" changed after 7 November, we computed the average Flesch-Kincaid grade level (Kincaid et al. 1975), which estimates an approximate (US) grade level indicating the readability of a document, for all tweets per day. To explore how individuals' writing changed before and after 7 November, we fit a statistical model to the daily distribution of tweet lengths. This model assumed a stochastic generative process whereby individuals would author a tweet of random length $L$, drawn from a 2-parameter length distribution $f(L ; \mu, \sigma)$; if $\ell>L_{\max }$ (i.e., if the individual wrote a tweet longer than the perceived character limit, $L_{\max }=140$ or $L_{\max }=$ 280 ) they would "edit" their tweet such that the final message length was $L=L_{\max }-\ell$, where $\ell$ was drawn from a 1-parameter distribution $g(\ell ; \lambda)$. We modeled individuals' observed delay in adapting to the longer character length $L_{\max }$ through a parameter $p$ controlling the probability of using $L_{\max }=140$ or $L_{\max }=280$. We performed model selection using Akaike's information criterion (AIC) to determine the most appropriate distributions $f$ and $g$, and used Approximate Bayesian Computation (ABC) (e,g., Toni et al. 2009) to obtain posterior estimates of the parameters $\mu, \sigma, \lambda_{i}, p$.

\section{Results}

Figure 1 shows that the average Flesch-Kincaid reading grade clearly increased by approximately half a grade after the character limit change (indicated by the vertical line), with a change-point analysis (Lavielle 2005) showing a significant change in the standard deviation of the signal at this same time. Interestingly, despite the increased character length a substantial drop in average tweet reading level was observed around New Years Eve 2017. Future work could explore whether this is an annual trend or not, the language features driving the change, and cultural reasons underlying why this particular holiday (rather than, e.g., Christmas) exhibits such a stark decrease in linguistic complexity.

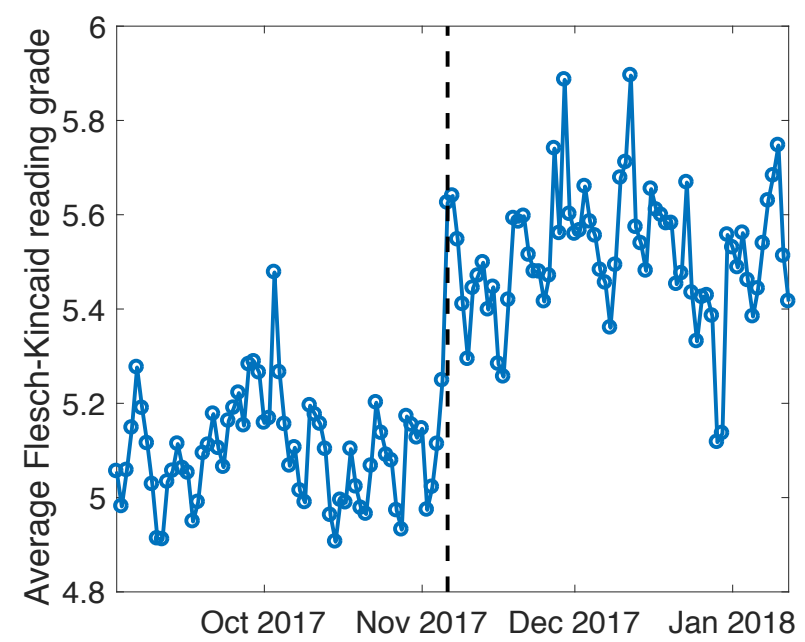

Figure 1: Average tweet Flesch-Kincaid reading level before and after Twitter's character limit change (vertical line). 
Consistent with the literature (Szell et al. 2015), AIC model selection on a range of message length distributions (Poisson, negative binomial, lognormal, Sichel) selected a lognormal distribution as the best model for the majority of the tweet length distributions, both before and after the character limit change. Figure 2 shows one such model fit, for 21 November 2017, 2 weeks after the change.

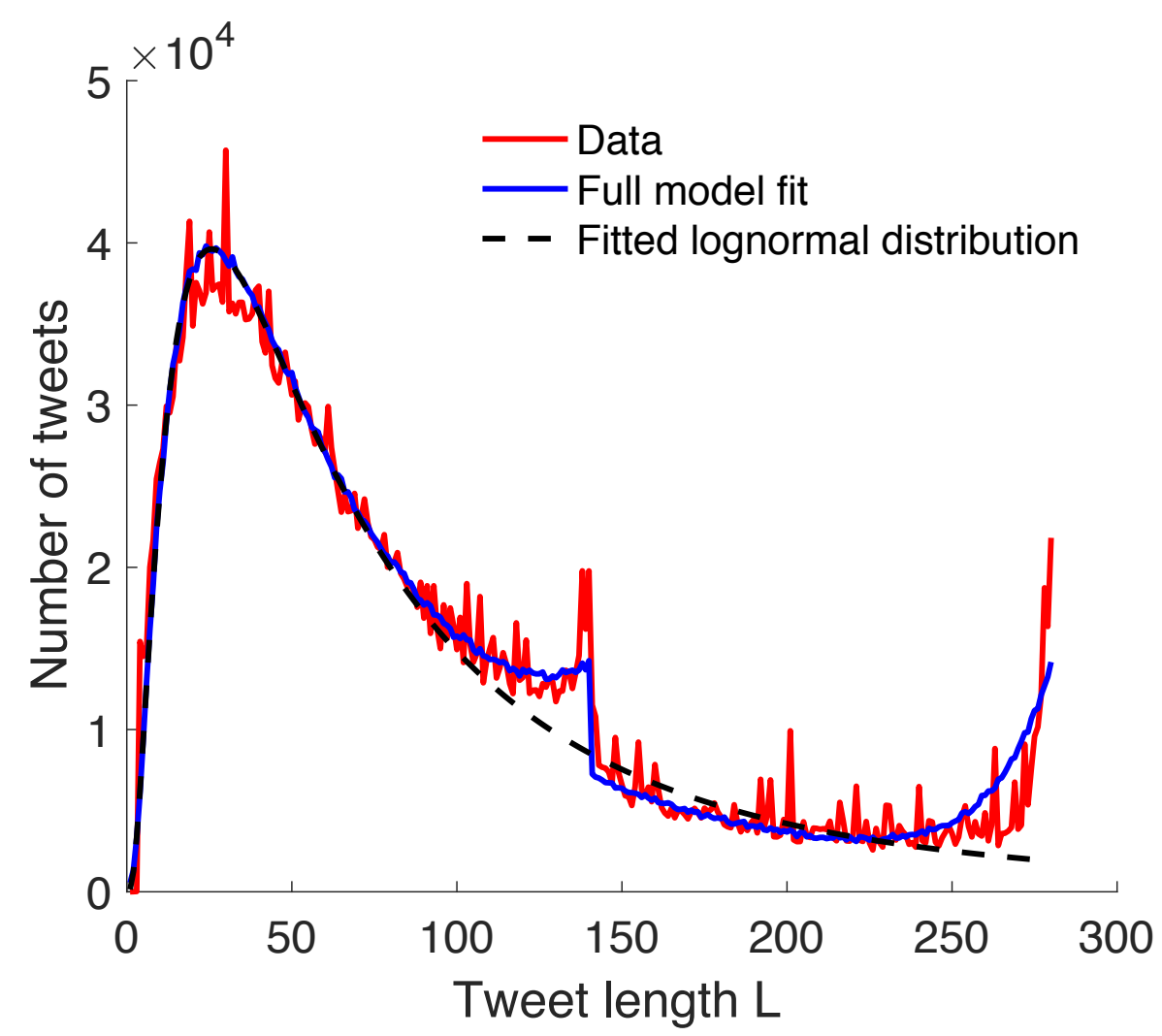

Figure 2: Model fits to the tweet length distribution.

The difference between the observed distribution of tweet lengths and the expected lognormal fits for (a) $100<\mathrm{L}<140$ and (b) $240<\mathrm{L}<280$ provides estimates of the proportions of individuals who either (a) unnecessarily self-edited their tweets based on a perceived maximum length of 140 characters, or (b) found that the new 280 character limit to still be insufficient and self-edited their tweet. These proportions are shown in Figure 3, for the period after 7 November 2017. 


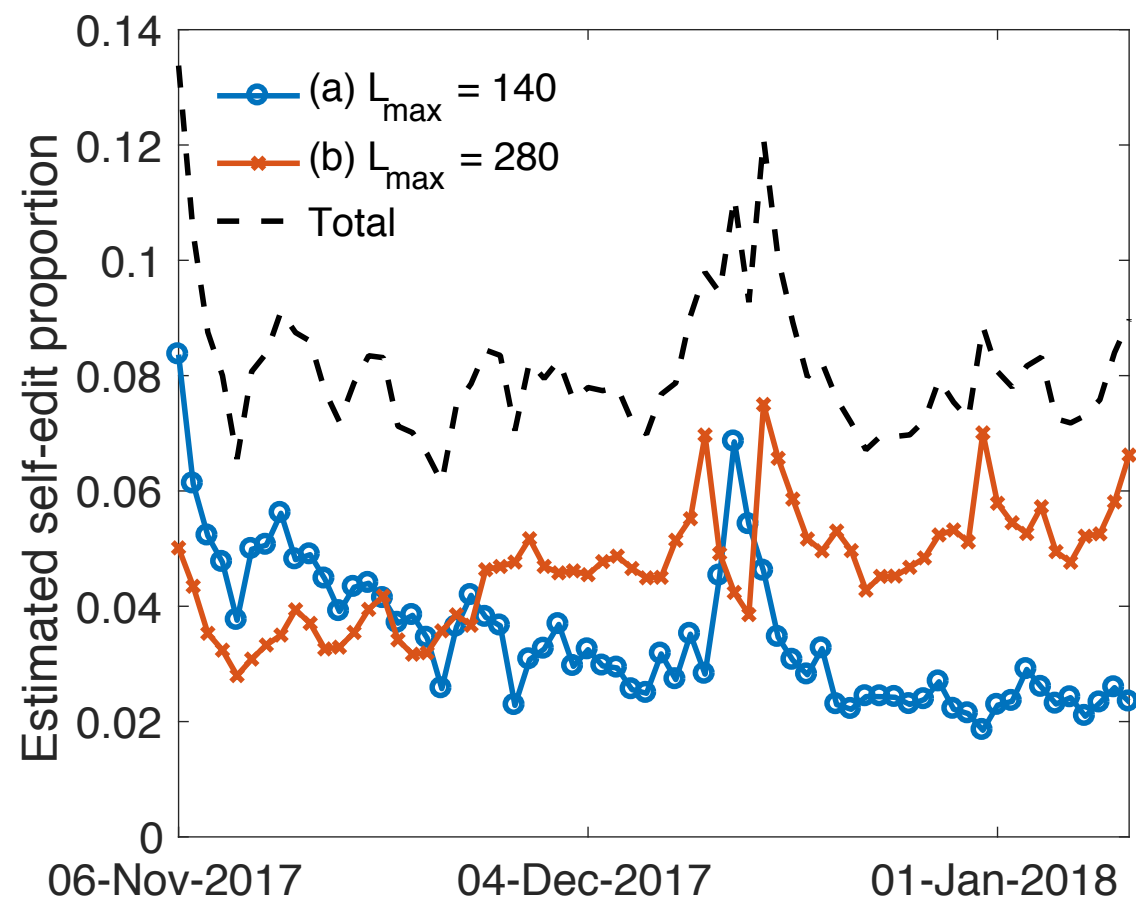

Figure 3: Estimated proportions of self-editors. Note that the overall proportion stays roughly constant.

We remark on a number of interesting findings revealed by this analysis. First, the estimated proportion of individuals apparently not realizing that the character limit increased after 7 November 2017 is surprisingly high, with over $5 \%$ of the Twitter-using population continuing to exhibit behavior consistent with a perceived 140-character limit a week after the change. Indeed, it appears to take at least 3 weeks for this proportion to equilibrate, with still a slight downward trend in January 2018. This suggests a surprisingly long response time for this population, with individual behavior taking weeks or possibly months to adjust to changes in the medium.

Furthermore, the decrease in this proportion is approximately balanced by an increase in the estimated proportion of users self-editing their tweets back from 280 characters. This produces a roughly constant proportion of around $8 \%$ of the population being "dissatisfied" with the increased character limit. While this has clearly decreased from the dissatisfaction level of $22 \%$ reported by Szell et al. (2014) for the previous $140-$ character limit, it is substantially more than the $1.7 \%$ they estimated if the character limit were increased to $L_{\max }=256$. This, coupled with the change in average Flesch-Kincaid reading grade (Figure 1), suggests a shift in individual behavior induced by the exogenous change to the medium of communication.

\section{Discussion}

These results provide the first empirical evidence for a shift in individual communication and linguistic evolution on the Twitter online social network after the 7 November 2017 character limit increase. While limited to a single platform, our results are consistent with the findings of other authors performing similar studies across multiple online media (Szell et al. 2015, Sobkowicz et al. 2013). 
In particular, the agreement we found between the data and a lognormal distribution (Figure 2) is striking. What is it about Twitter (and other online communication media) that appears to give rise to this distribution, regardless of constraints on message length? Gros et al. (2012) suggest that neurophysiological constraints in perceiving multiple forms of information limit the ability to produce new information, which ultimately gives rise to multimedia files being lognormally distributed. Sobkowicz et al. (2013) provide experimental evidence for internet post lengths, that perceived differences in the times taken to assimilate information and then write new content leads to lognormal distributions, a consequence of the Weber-Fechner law in psychology. Our results provide further observational evidence for this theory.

Finally, we remark that a key ingredient for this analysis was the use of generative mathematical models, coupled with efficient Bayesian parameter estimation algorithms (ABC) to quantify the not-directly-observable proportions of "dissatisfied" individuals on Twitter. We argue that mathematical models present to the modern Internet Researcher a powerful alternative exploratory methodology for "Big" social media datasets, and that such computational methods empower a deeper understanding of the human and social processes underlying the patterns observed in large-scale Internet research.

\section{References}

Gros, C., Kaczor, G., \& Marković, D. (2012). Neuropsychological constraints to human data production on a global scale. European Physical Journal B, 85(1), 28.

Kincaid, J. P., Fishburne, R. P. Jr, Rogers, R. L., \& Chissom, B. S. (1975). "Derivation of new readability formulas (Automated Readability Index, Fog Count and Flesch Reading Ease Formula) for Navy enlisted personnel". Research Branch Report 875, Millington, TN: Naval Technical Training, U. S. Naval Air Station, Memphis, TN.

Lavielle, M. (2005). Using penalized contrasts for the change-point problem. Signal Processing. 85(August), 1501-1510.

Rosen, A. (2017). Tweeting made easier. Twitter developer blog, 7 November 2017. URL: https://blog.twitter.com/official/en us/topics/product/2017/tweetingmadeeasier.html

Sobkowicz, P., Thelwall, M., Buckley, K., Paltoglou, G., \& Sobkowicz, A. (2013). Lognormal distributions of user post lengths in Internet discussions - a consequence of the Weber-Fechner law? EPJ Data Science, 2(1), 2.

Szell, M., Grauwin, S., \& Ratti, C. (2014). Contraction of online response to major events. PLOS ONE, 9(2), e89052.

Toni, T., Welch, D., Strelkowa, N., Ipsen, A., \& Stumpf, M. P. H. (2009). Approximate Bayesian computation scheme for parameter inference and model selection in dynamical systems. Journal of The Royal Society Interface, 6(July), 187-202. 